\section{Frecuencia de ateromas en radiografías panorámicas de pacientes mayores de 40 años con enfermedad periodontal atendidos en una Clínica Dental Universitaria}

Quiñónez P, Calderón V, Quintana M. Frecuencia de ateromas en radiografías panorámicas de pacientes mayores de 40 años con enfermedad periodontal atendidos en una Clínica Dental Universitaria. Rev Estomatol Herediana. 2006; 16 (2) : 110 - 114.

RESUMEN

El propósito del presente estudio fue determinar la frecuencia de ateromas en radiografías panorámicas de pacientes mayores de 40 años con enfermedad periodontal atendidos en la Clínica Dental de la Facultad de Estomatología Roberto Beltrán Neira de la Universidad Peruana Cayetano Heredia durante el período 2000 al 2004. La muestra consistió en 217 radiografías panorámicas elegidas por muestreo no probabilistico. El análisis de las variables se hizo usando tablas de frecuencia y se usó la prueba de Chi cuadrado para la evaluación de la significancia. Los resultados mostraron que la frecuencia de ateromas observados en las radiografías panorámicas de pacientes con enfermedad periodontal fue de $17,1 \%$. La frecuencia de ateromas fue mayor en el rango de edad de 40 a 59 años y mayor en el sexo femenino.

Palabras clave: ENFERMEDADES DE LAS ARTERIAS CARÓTIDAS. epidemiología / PERIODONTITIS.

The frequency of atheromas in panoramic radiographs of pacientes with periodontal disease, 40 year and older attending the University Dental Clinic

ABSTRACT

The aim of the present study was to determine the frequency of atheromas in panoramic radiographic in patients 40 years and older with periodontal diseases attending the Dental School "Roberto Beltrán Neira" in the Universidad Peruana Cayetano Heredia, during the period 2000 and 2004. The sample consisted of 217 random panoramic radiographic with no systematically selected subjects. Variables were analyzed using frequency tables and the chisquare test. Results showed the frequency of atheromas was of $17.1 \%$ and the highest number of atheromas appeared between the ages of 40 and 59 years, mostly of feminine sex.

Key words: CAROTID ARTERY DISEASES. epidemilogy / PERIODONTITS.
Patricia Quiñónez Bartra ${ }^{1}$ Víctor Calderón Ubaqui ${ }^{2}$ Martín Quintana del Solar ${ }^{3}$

Cirujano Dentista

Docente del Departamento Académico de Medicina, Cirugía y Patología Oral.

${ }^{3}$ Docente del Departamento Académico de Clínica Estomatológica.

Facultad de Estomatología. Universidad Peruana Cayetano Heredia.

\section{Correspondencia}

Patricia Quiñónez Bartra

Av. Tomas Marsano 2875. Dpto 504 - Lima 33, Perú Teléfono: (51-1) 2714879

e-mail: apatriciaqb @ hotmail.com

Recibido : 10 de noviembre del 2005

Aceptado : 10 de marzo del 2007

\section{Introducción}

La enfermedad periodontal es una de las infecciones crónicas más prevalente del ser humano. En la literatura no existen estudios epidemiológicos de aterosclerosis (ateroma) diagnosticados a través de la radiografía panorámica de pacientes con enfermedad periodontal (1-3).

Actualmente existe controversia sobre su posible origen exógeno y su transmisión intrafamiliar; la expresión clínica de la periodontitis varía entre los individuos, es de naturaleza cíclica y episódica con etapas de exacerbación, remisión y puede presentarse de manera localizada o generalizada cuando están afectados la mayoría de los dientes (3).

En un estudio retrospectivo, Lagervall, Jansson, Bergstrom (4), encontraron que una amplia proporción de pacientes con enfermedad periodontal son afectados por enfermedades sistémicas (5-8). Entre las enfermedades más prevalentes encontraron: la alergia (6), enfermedades cardiovasculares (6-8), enfermedades endocrinas (6-8), enfermedades vasculares (6) y enfermedades ortopédicas incluyendo artritis (6) y artritis reumatoidea (9).

Todos estos estudios en donde relacionan a la enfermedad periodontal con otras enfermedades sistémicas varían en el diseño, forma de medir la salud periodontal y procedimientos para obtener datos de salud médica (4).

La enfermedad cardiovascular es la responsable del 29\% de las muertes alrededor del mundo (10-12).
Uno de los principales componentes de esta enfermedad es la aterosclerosis $(13,14)$.

La aterosclerosis es una enfermedad degenerativa que se manifiesta por placas fibrosas que se depositan en la pared interna de las arterias disminuyendo el lumen del vaso y provocando pérdida de su elasticidad. A este acúmulo se le conoce como ateroma que con el tiempo se calcifica y se hace visible en los estudios por imágenes (15).

La teoría actual de la patogenia de la aterosclerosis establece que las lesiones se inician como respuesta a alguna forma de lesión del endotelio arterial producidas por diferentes factores (16), incluyendo: lípidos de baja densidad (LDL), radicales libres, hemocisteína, reacciones 
autoinmunes e infecciones microbianas $(14,17)$.

Los ateromas se forman en las arterias elásticas (aorta, carótida e ilíaca) $\mathrm{y}$ en arterias musculares grandes y medianas (coronarias y poplíteas). Como consecuencia de esto ocurre menor aporte sanguíneo y de oxígeno en los tejidos distales (16).

Los ateromas son mas frecuentes en varones mayores de 50 años y los factores que influyen en su formación son: hipertensión, consumo de tabaco, diabetes, hipercolesteremia, obesidad, tipo de vida sedentaria, consumo de alcohol, aumento de triglicéridos y antecedentes de ataques transitorios isquémicos $(11,14,18)$. Si bien la formación de ateromas es mayor en personas de 50 años, las estrías grasas se incrementan a partir de los 18 años y el 2\% de la población adulta de los EEUU sufre aterosclerosis sintomática de las extremidades inferiores con una relación de varones/mujeres de 2:1 (16).

Varios estudios epidemiológicos de casos controles han evaluado el riesgo de la presencia de enfermedad periodontal sobre la enfermedad cardiovascular (4,3,14,19-21).

Los primeros en llamar la atención fueron Mackenzie y Millard en 1963 (22) sobre una posible asociación entre enfermedad periodontal y cardiocascular al observar que pacientes no diabéticos con aterosclerosis presentaban mayor pérdida ósea respecto a controles de pacientes sanos.

Matilla et al en 1989 y 1993 (2325) presentaron informes que sirvieron de base para establecer las medidas de riesgo de enfermedad periodontal sobre la ocurrencia del infarto agudo del miocardio y la aterosclerosis .

De Stephano et al en 1996 (26) confirmaron la relación de estudios anteriores para enfermedad coro- naria incluyendo el infarto del miocardio no fatal, la angina de pecho y el infarto cerebral.

Genco et al (27), estudiaron indígenas americanos de la reserva de Gila River quienes presentaban un bajo nivel de tabaquismo con una alta tasa de diabetes. Este estudio de seguimiento longitudinal por quince años mostró una alta asociación entre enfermedad periodontal y enfermedad cardiovascular en ausencia de tabaquismo, siendo este un factor de riesgo para ambas enfermedades.

Matilla et al (23-25), De Stephano(26), Beck et al (28) ajustaron los riesgos relativos con análisis de represión logística y establecieron que estas asociaciones son independientes de edad, colesterol total, triglicéridos, hipertensión, diabetes (DBTs), índice de masa corporal, actividad física y consumo de tabaco.

La radiografía panorámica es un método simple y conveniente para obtener una perspectiva general de las arcadas dentarias (30-32). La radiografía panorámica diagnostica la enfermedad periodontal a través del grado de reabsorción ósea a nivel de crestas alveolares (17), aportan un bosquejo radiográfico general informativo sobre la distribución y gravedad de la destrucción ósea en la enfermedad periodontal, aunque el diagnóstico periodontal y la planeación terapéutica exigen una serie intrabucal completa $(2,29)$.

En el caso del diagnóstico radiográfico de los ateromas es posible cuando éstos están parcialmente calcificados a nivel de las arterias carótidas primitivas o en la bifurcación de las mismas (18)

Dos reportes de casos y un pequeño estudio (10 pacientes) publicados en la literatura dental han mostrado que las calcificaciones de carótidas en las radiografías pano- rámicas están asociadas con grados variables de estenosis. El grado de estenosis en la mayoría de pacientes con calcificaciones de carótidas observados en radiografías panorámicas permanece desconocido como lo es su pronóstico para ocurrencias cerebrovasculares y cardiovasculares $(12,33,34)$.

Radiológicamente la imagen del ateroma puede aparecer como una masa radiopaca nodular de 1.5 a 4 cms de diámetro o como dos líneas verticales inferiores localizadas a nivel del ángulo de la mandíbula o posterior e inferior al ángulo de la mandíbula en una angulación de 45 grados, por encima del hueso hioides y posterior a las vértebras C3- C4 (11, 18, 35, 36). La bifurcación de la carótida es el sitio usual para la calcificación pero puede estar ubicado más hacia abajo (11).

Las radiografías no determinan con exactitud la localización y el grado de oclusión que causan los ateromas en los vasos. Para ello existen técnicas invasivas y no invasivas, como el estudio doppler (ultrasononografía), angiografía y análisis espectrales que determinan con exactitud el diámetro del lumen del vaso $(17,18,37)$. Se unen a éstos las tomografías computarizadas y la resonancia magnética $(11,17$, 18, 19).

\section{Material y métodos}

El estudio fue de tipo transversal y descriptivo. Se tomaron 4,000 Historias Clínicas de pacientes adultos atendidos en la Clínica Estomatológica de la Universidad Peruana Cayetano Heredia durante el período de enero 2000 - diciembre 2004. Mediante un muestreo no probabilístico, se seleccionaron 217 radiografías panorámicas de pacientes con diagnóstico estomatológico de enfermedad periodontal. Las radiografías fueron 
analizados por un especialista de radiología oral que cuenta con más de cinco años de experiencia utilizando un negatoscopio, con intensidad de luz uniforme y constante, mediante visión directa complementada con la ayuda de una lupa con una magnificación de 3x2 de diámetro. Los resultados fueron sometidos a análisis univariado de la variable edad obteniendose una media y desviación estándar. Con la variable ateroma y sexo se obtuvo frecuencias y porcentajes. Se realizó el análisis bivariado mediante tablas de doble entrada. Se determinó la asociación de las variables de estudio mediante la prueba de Chi cuadrado a un nivel de significancia de 0,05. Se utilizó el programa SPSS versión 11.

\section{Resultados}

Se muestra el número y el porcentaje de ateromas en 217 pacientes con enfermedad periodontal, encontrandose que el $17,1 \%$ presenta ateromas.

La frecuencia de ateromas es mayor en pacientes con el rango de edades entre 40 a 49 años y de 50 a 59 años, encontrándose 10 ateromas para ambos, y menor en el rango de edad mayores a 70 años, encontrándose 8 ateromas de los 37 ateromas diagnosticados. Pero existe un mayor porcentaje en la presencia de ateromas en el rango de edad de 70 años a mas, con un $23 \%$, a diferen- cia del $13 \%$ que encontramos en el rango de 50 a 59 años (Tabla 1). La media de los pacientes evaluados fue de 57.64 años con una desviación estándar de 10.67.

Encontramos que la frecuencia de ateromas es mayor en el sexo femenino (25 ateromas) con respecto al masculino (12 ateromas).

Encontramos que no existe una asociación estadísticamente significativa entre ateromas y la edad $(p=0.648)$. También no existe una asociación estadísticamente significativa entre ateromas y sexo $(\mathrm{p}=$ 0.980).

\section{Discusión}

Desde hace muchos años, diversos estudios han venido reportando la detección de ateromas por medio de la radiografía panorámica con una prevalencia de 2 a $37 \%$ en diferentes condiciones. En nuestro estudio, de las 217 radiografías panorámicas evaluadas de pacientes con enfermedad periodontal, se encontraron 37 ateromas (17.1\%). Demostrando que es un hallazgo radiográfico poco prevalente. Estos resultados coinciden con el estudio de Friedlander et al, quienes encontraron una prevalencia del 21\% (12).

El diagnóstico radiográfico de los ateromas es posible cuando éstos están parcialmente calcificados a nivel de las arterias carótidas primitivas o en la bifurcación de las mismas (18). Por lo tanto la radiografía

Tabla 1. Presencia y ausencia de ateromas según rango de edades.

\begin{tabular}{lllrlr}
\hline Rango de edades & \multicolumn{2}{c}{ Ausente } & \multicolumn{2}{c}{ Presente } & Total \\
& $\mathrm{n}$ & $(\%)$ & $\mathrm{n}$ & $(\%)$ & $\mathrm{n}$ \\
\hline 40 a 49 años & 44 & $(81)$ & 10 & $(19)$ & 54 \\
50 a 59 años & 65 & $(87)$ & 10 & $(13)$ & 75 \\
60 a 69 años & 44 & $(83)$ & 9 & $(17)$ & 53 \\
70 a mas & 27 & $(77)$ & 8 & $(23)$ & 35 \\
Total & 180 & & 37 & & 217 \\
\hline
\end{tabular}

panorámica no sólo sirve para evaluar dientes y maxilares, si no también para identificar alteraciones en la región blanda del cuello, como son las calcificaciones en la arteria carótida (37).

Es necesario ampliar los exámenes auxiliares para un mejor diagnóstico del ateroma en cuanto a localización, extensión y grado de oclusión que éstos causan, Ohba et al(37) concluyeron en un estudio realizado el 2003 que la radiografía panorámica detecta la presencia de tales placas calcificadas con limitación, ya que el nivel de bifurcación de las arterias carótidas, donde son visibles los ateromas, están situados en el lado derecho entre la C3 y C4 mientras que el izquierdo entre la $\mathrm{C} 4$ y C5 donde no se proyecta totalmente las radiografías panorámicas; por lo tanto se especula que los ateromas en el lado izquierdo son menos detectados a menudo que aquellos en el lado derecho.

Los ateromas carotídeos en nuestra población de estudio tuvieron aparencias morfológicas similares; variando desde calcificaciones radiopacas simples a múltiples; estuvieron localizados dentro de los tejidos blandos a nivel del cuello, aproximadamente $2.5 \mathrm{~cm}$ debajo y posterior al ángulo de la mandíbula. La presencia de calcificaciones en la carótida puede indicar el probable punto de oclusión, esta información debe estar incorporada en la Historia Clínica del paciente, para el conocimiento del médico (35). Para el diagnóstico diferencial se debe ampliar la anamnesis, haciendo un examen clínico mas profundo (36).

Con respecto a la edad y la presencia de ateromas, se encontró una mayor frecuencia de ateromas en el rango de 50 a 59 años (75 ateromas) $\mathrm{y}$ una menor frecuencia en el rango de 70 a más (35 ateromas). Sin em- 
bargo hay un mayor porcentaje de ateromas en el rango de edad de 70 a más (23\%), esto se debe a que se evaluó menor población en este rango de edad. No existiendo asociación con respecto a la edad. Esto se puede explicar a que este hallazgo es más prevalente en personas mayores. Estos resultados son similares a los encontrados por Lewis et al, en donde encontraron que la presencia de ateromas se encuentra en el rango de 55 a 59 años (11). Igualmente en le estudio de Friedlander et al encontró la presencia de ateromas en pacientes mayores de 65 años (12). En el estudio de Ohba et al, se encontró la presencia de ateromas en edades de 80 años (37). Es importante detectar el ateroma en pacientes mayores por que la arterioesclerosis es un proceso progresivo y puede conducir a ataques isquémicos transitorios o accidentes cerebrovasculares (37). Los hombres tienen dos veces más riesgo de un accidente cardiovascular que las mujeres de la misma edad (11). Los ateromas no desaparecen, son estables y progresan hacia una formación de hemorragias intramurales y calcificación de la íntima (16).

Con respecto al sexo y la presencia de ateromas, no existe asociación con respecto al sexo, ya que para ambos encontramos que la prevalencia fue la mismas (17 \%). Estos resultados son similares al estudio realizado por Ohba et al (37), el cual encontró que no hay asociación entre sexo y ateromas. Los factores de riesgo para la formación de estas placas es igual para ambos sexos (11).

\section{Conclusiones}

La frecuencia de ateromas fue mayor en el rango de edad de 40 a 59 años y en el sexo femenino.

No existió asociación entre la presencia de ateromas con la edad y sexo.

\section{Referencias Bibliográficas}

1. López FR, Oyarzún MM, Naranjo CC. Asociación entre periodontitis y enfermedad cardiovascular. Rev Méd Chile. 2000; 128(11):1295-6.

2. Suarez-Cunqueiro MM, Duker J, Liebehenschel N, Schön R, Schmelzeisen R. Calcification of the branches of the external carotid artery detected by panoramic radiography: a case report. Oral Surg Oral Med Oral Pathol Oral Radiol Endod. 2002; 94(5):636-40.

3. Olry R, Lellouch A. The arterial system of the Japanese anatomist Buntaro Adachi. Hist Sci Med. 2003; 37(1):89-94

4. Lagervall $M$, Jansson L, Bergström J. Systemic disorders in patients with periodontal disease. J Clin Periodontol. 2003;30(4):293-9.

5. Brasher WJ, Rees TD. Systemic conditions in the management of periodontal patients. J Periodontol. 1970;41(6):349-52.

6. Rees TD, Brasher WJ. Incidence of certain systemic conditions among patients presenting for periodontal treatment. J Periodontol. 1974; 45(9):669-71.

7. Nery EB, Meister F Jr, Ellinger RF, Eslami A, McNamara TJ. Prevalence of medical problems in periodontal patients obtained from three different populations. J Periodontol. 1987; 58(8):564-8.

8. Peacock ME, Carson RE. Frequency of self-reported medical conditions in periodontal patients. J Periodontol. 1995; 66(11):10047.

9. Mercado F, Marshall RI, Klestov AC, Bartold PM. Is there a relationship between rheumatoid arthritis and periodontal disease? J Clin Periodontol. 2000; 27(4): 267-72.

10. Vinereanu D. Risk factors for atherosclerotic disease: present and future. Herz. 2006; 31(Suppl 3):5-24.

11. Lewis DA, Brooks SL. Cartoid artery calcification in a general dental population : a retrospective study of panoramic radiographs. Gen Dent. 1999; 47(1):98-103.

12.Friedlander AH, Baker JD. Panoramic radiography: an aid in detecting patients at risk of cerebrovascular accident. J Am Dent Assoc. 1994; 125(12):1598-603.

13. Carter LC, Tsimidis K, Fabiano J. Carotid calcifications on panoramic radiography identify an asymptomatic male patient at risk for stroke. A case report. Oral Surg Oral Med Oral Pathol Oral Radiol Endod. 1998;85(1):119-22.

14.Cuspidi C, Meani S, Valerio C, Fusi V, Sala C, Zanchetti A, Mancia G. Carotid atherosclerosis and cardiovascular risk stratification : role and costeffectiveness of echo-Doppler examination in untreated essential hypertensives. Blood Press. 2006;15(6):333-9.

15. Martinez JL, Penna M. Influences of changes in calcium concentrations, cocaine and clonidine on the cardiac effect of acetaldehyde in rat isolated atria. Gen Pharmacol. 1997; 29(2):281-4.

16.Canseco-Avila LM, JerjesSanchez C, Ortiz-Lopez R, Rojas-Martinez A, GuzmanRamirez D. Fibrinogen. Cardiovascular risk factor or marker?. Arch Cardiol Mex. 2006; 76(Suppl 4):S158-72.

17. Rossi M, Galetta F, Franzoni F, Antonelli A, Santoro G. Cardiovascular remodelling in patients 
with sub-clinical hypothyroidism. Minerva Cardioangiol. 2006; 54(6):807-10

18. Manzi FR, Boscolo FN, de Almeida SM, Haiter Neto F. Panoramic radiography as an auxiliary in detecting patients at risk for cerebrovascular accident (CVA): a case report. J Oral Sci. 2003;45(3):177-80.

19. Janket SJ, Baird AE, Chuang SK, Jones JA. Meta-analysis of periodontal disease and risk of coronary heart disease and stroke. Oral Surg Oral Med Oral Pathol Oral Radiol Endod. 2003;95(5):559-69.

20. Seymour RA, Preshaw PM, Thomason JM, Ellis JS, Steele JG. Cardiovascular diseases and periodontology. J Clin Periodontol. 2003;30(4):279-92.

21. Madianos PN, Bobetsis GA, Kinane DF. Is periodontitis associated with an increased risk of coronary heart disease and preterm and/or low birth weight births? J Clin Periodontol. 2002;29(Suppl 3):22-36

22. Mackenzie RS, Millard HD. Interrelated effects of diabetes, arteriosclerosis and calculus on alveolar bone loss. J Am Dent Assoc 1963: 66- 92.

23. Mattila KJ. Dental infections as a risk factor for acute myocardial infarction. Eur Heart J. 1993;14(Suppl K):51-3.

24. Mattila KJ, Nieminen MS, Valtonen VV, Rasi VP, Kesaniemi YA, Syrjala SL, Jungell PS, Isoluoma $\mathrm{M}$,
Hietaniemi K, Jokinen MJ. Association between dental health and acute myocardial infarction. Br Med J. 1989; 298(6676):779-81.

25. Mattila KJ, Valle MS, Nieminen MS, Valtonen VV, Hietaniemi KL. Dental infections and coronary atherosclerosis. Atherosclerosis. 1993;103(2): 205-11.

26. DeStefano F, Anda RF, Kahn HS, Williamson DF, Russell CM. Dental disease and risk of coronary heart disease and mortality. Br Med J. 1993; 306(6879):688-91.

27.Genco RJ, Grossi SG, Ho A, Nishimura F, Murayama Y. A proposed model linking inflammation to obesity, diabetes, and periodontal infections. J Periodontol. 2005 Nov;76(11 Suppl): 2075-84.

28.Beck J, Garcia R, Heiss G, Vokonas PS, Offenbacher S. Periodontal disease and cardiovascular disease. J Periodontol. 1996;67(10 Suppl):1123-37.

29. Lang L. Congress Passes 2006 NIH Reform Act. Gastroenterology. 2007;132(2):474-5.

30. Herzberg MC, Weyer MW. Dental plaque, platelets, and cardiovascular diseases. Ann Periodontol. 1998;3(1):151-60.

31.Deshpande RG, Khan M, Genco CA. Invasion strategies of the oral pathogen porphyromonas gingivalis : implications for cardiovascular disease. Invasion Metastasis. 1998-1999;18(2):5769.
32. Mattila KJ, Valtonen VV, Nieminen MS, Asikainen S. Role of infection as a risk factor for atherosclerosis, myocardial infarction, and stroke. Clin Infect Dis. 1998;26(3):719-34.

33. Handa N, Matsumoto M, Maeda $\mathrm{H}$, Hougaku H, Kamada T. Ischemic stroke events and carotid atherosclerosis. Results of the Osaka Follow-up Study for Ultrasonographic Assessment of Carotid Atherosclerosis (the OSACA Study). Stroke. 1995; 26(10):1781-6.

34. Almog DM, Illig KA, Khin M, Green RM. Unrecognized carotid artery stenosis discovered by calcifications on a panoramic radiograph. J Am Dent Assoc. 2000;131(11):1593-7.

35.Friedlander AH, Lande A. Panoramic radiographic identification of carotid arterial plaques. Oral Surg Oral Med Oral Pathol. 1981;52(1):102-4.

36. Cohen SN, Friedlander AH, Jolly DA, Date L. Carotid calcification on panoramic radiographs: an important marker for vascular risk. Oral Surg Oral Med Oral Pathol Oral Radiol Endod. 2002;94(4):510-4.

37. Ohba T, Takata Y, Ansai T, Morimoto Y, Tanaka T, Kito S, Awano S, Akifusa S, Takehara T. Evaluation of calcified carotid artery atheromas detected by panoramic radiograph among 80year-olds. Oral Surg Oral Med Oral Pathol Oral Radiol Endod. 2003;96(5):647-50. 